\title{
Fullwave Volumetric Maxwell solver using Conduction Modes
}

\author{
Salvador Ortiz ${ }^{*}$ \\ Roberto Suaya $^{\dagger}$
}

\begin{abstract}
We present a gridless method for solving the interior problem for a set of conductors in an homogeneous dielectric, at sufficiently high frequencies, valid for conductor lengths that are not small compared to the minimum wavelength, and transverse dimensions that are large compared to the skin depth. For IC applications, we cover the regime $10-100 \mathrm{GHz}$ and the inclusion of all relevant wire dimensions. We decompose the Electromagnetic field in terms of the eigenfunctions of the Helmholtz equation for three dimensional current distributions inside the conductors. Using a relatively small number of modes per conductor we obtain results comparable to filament or mesh decompositions using a much larger dimensionality for the resulting linear problem. The method is an extension to the fullwave regime of a method introduced in [1].
\end{abstract}

\section{INTRODUCTION}

Interconnect effects in signal propagation and noise analysis pose ever increasing demands on electromagnetic solvers. Maximum frequencies attainable with CMOS technology at $65 \mathrm{~nm}$ mode are in the neighborhood of $40 \mathrm{GHz}$. The frequency range $(10-100) \mathrm{GHz}$ is one where proximity and skin effect become fully developed. Signals propagating at these frequencies, on $C u$ wires with transverse dimensions in the order of $1 \mu \mathrm{m}$ penetrate the conductors only a fraction of its cross section. In this regime, frequencies are too high for considering the currents to be uniform over the cross section, and too low for considering them as purely superficial. Global interconnect and some intentional passive devices have lengths comparable to the minimal wavelength of light in the medium. Good and efficient computational schemes have been developed in EM applications for the very long as well as for the very short wavelength regimes [2]. In the IC domain up to frequencies of $10 \mathrm{GHz}$ the long wavelength regime suffices to study EM phenomena. Above these frequencies we enter the intermediate wavelength scale which

\footnotetext{
*Corresponding author: salvador_ortiz@mentor.com, S.Ortiz is a Phd candidates at Université Joseph Fourier, Grenoble, France.

${ }^{\dagger}$ S.Ortiz, and R.Suaya are with Mentor Graphics Ireland French Branch, 180 Av de l'Europe, 38334 St Ismier, France
}

Permission to make digital or hard copies of all or part of this work for personal or classroom use is granted without fee provided that copies are not made or distributed for profit or commercial advantage and that copies bear this notice and the full citation on the first page. To copy otherwise, to republish, to post on servers or to redistribute to lists, requires prior specific permission and/or a fee.

ICCAD’06, November 5-9, 2006, San Jose, CA

Copyright 2006 ACM 1-59593-389-1/06/0011...\$5.00 has been much less explored. Seminal contributions to high performance field solvers in the long wavelength domain, developed in the early 1990's, have been widely used in industry, namely the quasi-electrostatic (EQS) Capacitance solver FastCap [3] and the quasi-magnetostatic (MQS) $R L$ solver FastHenry [4]. In the intermediate wavelength scale, some recent contributions $[5,6]$ are today's reference in the field. Both teams use integral surface representations for the EM field. The conditioning number of the resulting linear system is an open question and a source of concern. The UIUC team uses different mesh decomposition of the surfaces depending on the frequency range [6], while the MIT team is currently revisiting a volume formulation, in part driven by this concern [7].

The computational demands associated to this new generation of EM solvers is an an open area for research and development. Motivated by this concern, we were attracted by the work of [1] who introduced conduction modes as an alternative to the filament decomposition for examining skin effect influence on $R L$ computations. Their work falls entirely within the MQS approximation and as such valid for long wavelengths. A major impact of that paper comes from the fact that few modes were needed to reproduce the FastHenry results with good accuracy in the presence of significant skin effect. The computational savings resulting from this approximation vis a vis a filament decomposition within FastHenry were significant. With this result in mind we studied the extension of the volumetric conduction mode treatment to the fullwave domain, in the presence of wire lengths comparable to the minimum wavelength. In this paper we are reporting our first conclusions.

In generalizing the method of conduction modes basis functions, from the $R L-\mathrm{MQS}$ approach to the $R L C$ - fullwave domain, the first approximation we dropped is that of unidimensional current distributions, with the direction being that of the gradient of the applied potential. The unidimensional nature of the currents and magnetic vector potential is prevalent in quasistatic analysis and cannot be kept in the fullwave regime. Transverse currents are a necessary ingredient and are responsible for the deposition of charges on the conductors' non-contact surfaces. These surface charges give rise to capacitance effects between conductors, and are hereby treated within the same framework as the one used to describe resistive and inductive effects. Their inclusion impacts on the choice of the boundary conditions on the conductor surfaces, on a full solver. In essence, the $\mathbf{E}$ field on the non contact surfaces develops a small tangential component, as expected for a non perfect conductor [8].

We use a mixed potential integral equation (MPIE) approach to describe the electric field $\mathbf{E}$ in terms of the gradient of electric scalar potential and the time derivative of the magnetic vector potential. To make room for the length scales comparable to the minimum wavelength we modify the Green function of the problem in the presence of a homogeneous dielectric from the Coulomb Green 
function to the fullwave Green function.

In the following two sections we present the equations for the linear system. The fundamental results later used in this work are contained in equations (5), (9) and (11). In section 4, we describe the $R L$ conduction mode parametrization and the $R L C$ generalization. In section 5 we give a brief overview of some of the numerical challenges encountered in performing the numerical integrations in (5). In section 6, we give a couple of examples, on the the first one we examine the stability of the method in moving toward the quasistatic regime. We verify that in fact we recover FastHenry results for small conductor lengths compared to the minimum $\lambda$. We next examine a simple fullwave electromagnetic problem and encounter the expected resonances not present in the quasistatic treatments. We compare our results to FastImp, and to the separated quasistatic approach resulting from merging the impedance computations from $R L$ with the $C$ results from FastCap.

\section{CHOICE OF LINEAR SYSTEM}

We start from a formal solution to the three dimensional Maxwell's equations. We work in the Lorenz gauge, with the conductors immersed in a homogeneous lossless dielectric, incorporate Ohms law and the continuity equation resulting from charge conservation, and perform our computation on the Fourier representation. We use a MPIE representation of the field $\mathbf{E}(\mathbf{r})$ in terms of the gradient of the scalar electric potential $\phi$ and the time derivative of the magnetic vector potential $\mathbf{A}(\mathbf{r})$. The unknown quantities are the currents in the interior of the conductors, and the charges on their surfaces, which are governed by the integral equations:

$$
\begin{aligned}
\frac{\mathbf{J}(\mathbf{r})}{\sigma}+\frac{j \omega \mu_{0}}{4 \pi} \int_{V} \mathbf{J}\left(\mathbf{r}^{\prime}\right) \frac{e^{-j k_{0}\left|\mathbf{r}-\mathbf{r}^{\prime}\right|}}{\left|\mathbf{r}-\mathbf{r}^{\prime}\right|} \mathrm{d} \mathbf{r}^{\prime} & =-\nabla \phi(\mathbf{r}) \\
\frac{1}{4 \pi \varepsilon} \int_{S} \rho\left(\mathbf{r}^{\prime}\right) \frac{e^{-j k_{0}\left|\mathbf{r}-\mathbf{r}^{\prime}\right|}}{\left|\mathbf{r}-\mathbf{r}^{\prime}\right|} \mathrm{d} \mathbf{r}^{\prime} & =\phi(\mathbf{r})
\end{aligned}
$$

with $\sigma$ the conductor's conductivity, $k_{0}=\omega \sqrt{\varepsilon \mu_{0}}=2 \pi / \lambda$ is the wave number, $V$ is the union of all conductor volumes $V_{i}$, and similarly for the surface $S$. Ohm's law has been used in replacing the total $\mathbf{E}$ in the conductor by $\mathbf{J}(\mathbf{r}) / \sigma$, and the common kernel $\exp \left(-j k_{0}\left|\mathbf{r}-\mathbf{r}^{\prime}\right|\right) /\left|\mathbf{r}-\mathbf{r}^{\prime}\right|$ is the Green function for this problem in the Lorenz gauge. In addition, the sources $\mathbf{J}$ and $\rho$ are related by the continuity equation:

$$
\begin{aligned}
& \nabla \cdot \mathbf{J}=0 \quad \text { inside the conductors } \\
& \mathbf{J} \cdot \hat{\mathbf{n}}=j \omega \rho \text { on conductors' }{ }^{\prime} \text { non }- \text { contact surfaces }
\end{aligned}
$$

as well as boundary conditions on the contact surfaces, $\phi(\mathbf{r}) \equiv$ $\phi_{S_{i}}$ at the electrical connexions to the conductors.

We expand the vector current density $\mathbf{J}$ and surface charge density $\rho$, in terms of two sets of functions $\mathbf{w}_{\mathbf{i}}(\mathbf{r}), i=1 \ldots n$ defined on the conductors volumes, and $v_{j}(\mathbf{r}), j=1 \ldots n$ defined on the conductors' surfaces. For compactness of the notation, we absorb into the single index $i$ the dependence on the conductor as well as the particular mode. Where it might lead to confusion we clarify the respective roles. The total number $n$ is the sum of modes over all conductors.

$$
\begin{array}{lr}
\mathbf{J}(\mathbf{r}) \approx \sum_{j} I_{j} \mathbf{w}_{\mathbf{j}}(\mathbf{r}) & \mathbf{r} \in V \\
\rho(\mathbf{r}) \approx \sum_{j} q_{j} v_{j}(\mathbf{r}) & \mathbf{r} \in S_{\text {non-contact }}
\end{array}
$$

To determine the coefficients $I_{i}, q_{j}$ on these expansions, we use the Galerkin method of moments, whereby we replace (3b) into (1a) and (1b), perform a functional inner product with $\mathbf{w}_{\mathbf{i}}(\mathbf{r})$ for (1a) and with $v_{j}(\mathbf{r})$ for (1b), with the requirement that the residuals be orthogonal to the basis functions. The resulting equations are represented by a $(2 n)^{2}$ linear system that can be written as:

$$
\left.\left[\begin{array}{c:c}
R+j \omega L & 0 \\
\hdashline 0 & P
\end{array}\right]\left(\begin{array}{c}
I \\
\hdashline q
\end{array}\right)=\left(\begin{array}{c}
-<\mathbf{w}_{i}, \nabla \phi> \\
\hdashline<v, \phi>
\end{array}\right)\right\} \begin{aligned}
& n \\
& \hdashline
\end{aligned}
$$

with the matrix elements given by:

$$
\begin{aligned}
R_{i j} & =\frac{1}{\sigma} \int_{V} \mathbf{w}_{i}^{*}(\mathbf{r}) \cdot \mathbf{w}_{j}(\mathbf{r}) \mathrm{d}^{3} r \\
L_{i j} & =\frac{\mu_{0}}{4 \pi} \int_{V} \int_{V} \mathbf{w}_{\mathbf{i}}{ }^{*}\left(\mathbf{r}^{\prime}\right) \mathbf{w}_{\mathbf{j}}(\mathbf{r}) \frac{e^{-j k_{0}\left|\mathbf{r}-\mathbf{r}^{\prime}\right|}}{\left|\mathbf{r}-\mathbf{r}^{\prime}\right|} \mathrm{d}^{3} r \mathrm{~d}^{3} r^{\prime} \\
P_{i j} & =\frac{1}{4 \pi \varepsilon} \int_{S} \int_{S} v_{i}^{*}\left(\mathbf{r}^{\prime}\right) v_{j}(\mathbf{r}) \frac{e^{-j k_{0}\left|\mathbf{r}-\mathbf{r}^{\prime}\right|}}{\left|\mathbf{r}-\mathbf{r}^{\prime}\right|} \mathrm{d}^{2} r \mathrm{~d}^{2} r^{\prime}
\end{aligned}
$$

with the subindices $i, j$ ranging from 1 to $n$, in the first two lines, and from $n+1$ to $2 n$ in the last one. The volume $V$ is the collection of all the conductors' volumes, whereas $S$ is the set of all the conductors' non-contact surfaces. We have a collection of three, four and six dimensional integrals to perform. The inner products appearing on the right hand side (rhs) of (4) are given by:

$$
\begin{aligned}
<\mathbf{w}_{i}, \nabla \phi> & =\int_{V} \nabla \phi \cdot \mathbf{w}_{i}^{*} \mathrm{~d}^{3} r \quad i=1 . . . n \\
<v_{i}, \phi> & =\int_{S} \phi v_{i}^{*} \mathrm{~d}^{2} r \quad i=n+1 . .2 n
\end{aligned}
$$

The effectiveness of the method will be assessed by the level of precision we can get using a relatively small number of modes for each conductor in $\mathbf{w}_{\mathbf{i}}(\mathbf{r})$ and $v_{i}(\mathbf{r})$, and the difficulty associated with computing (5).

In the quasistatic limit the two blocks in (4) are decoupled. Current distributions ( $R L$ extraction) and the charge distributions $(C$ extraction) are independently found. The choice of piecewise constant functions, vanishing outside the volume of a single filament of a conductor's volume for $\mathbf{w}_{\mathbf{i}}(\mathbf{r})$ and independently piecewise constant functions over surface panels for each conductor for $v_{i}(\mathbf{r})$, correspond to the model equations on FastHenry and FastCap, respectively. Boundary conditions for these two problems are different: for the $R L$ problem, they are tantamount to setting $\omega=0$ in the continuity equation $(2 b)$, whereas conductors are treated as equipotentials for the EQS problem. Moreover, in this nearly infinite wavelength approximation $\lambda \gg \ell$ (with $\ell$ the conductor's length) it is admissible to replace the phase factors by 1 inside the integrals in (5).

\section{RLC MIXED REPRESENTATION}

For the general $R L C$ extraction method, valid over a wider regime of length and frequencies, the coupling between (1a) and (1b) must be kept. Our goal in this section is to reduce the dimensionality of the linear system on (4) and express the unknowns solely in terms of currents. To this effect, we rewrite the linear system using Green's theorem: 

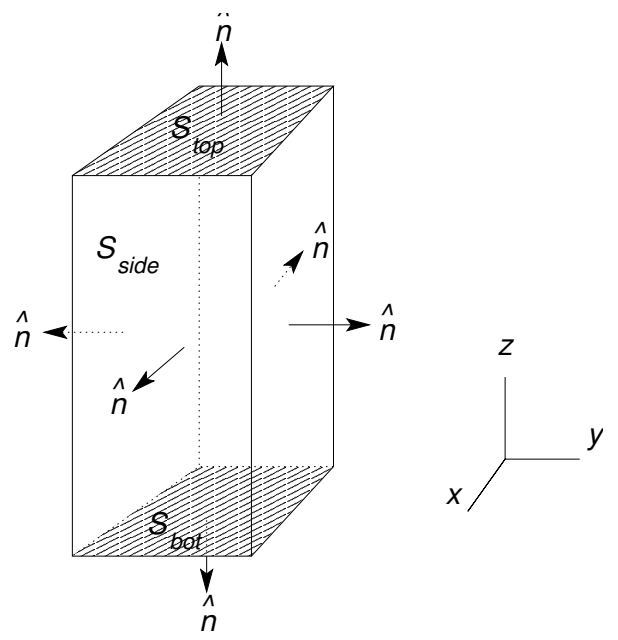

Figure 1: Surface $S(V)$ with its normal vectors $\hat{\mathbf{n}}$, decomposed into two contact surfaces $S_{t o p}, S_{b o t}$ and one non-contact surface $S_{\text {side }}$.

$$
\oiiint_{S\left(V_{i}\right)} \mathbf{F} \cdot \hat{\mathbf{n}} \mathrm{d}^{2} r=-\int_{V_{i}} \nabla \cdot \mathbf{F} \mathrm{d}^{3} r
$$

with $S\left(V_{i}\right)$ a closed surface that encloses volume $V_{i}$ (a single conductor) and we use it on $\mathbf{F}=\phi \mathbf{w}_{\mathbf{i}}^{*}$. We then use the identity $\nabla \cdot(\phi \mathbf{u})=\mathbf{u} \cdot \nabla \phi+(\nabla \cdot \mathbf{u}) \phi$ to obtain,

$$
\oiiint_{S\left(V_{i}\right)}\left(\mathbf{w}_{i}^{*} \cdot \hat{\mathbf{n}}\right) \phi \mathrm{d}^{2} r+\int_{V_{i}} \mathbf{w}_{i}^{*} \cdot \nabla \phi \mathrm{d}^{3} r+\int_{V_{i}}\left(\nabla \cdot \mathbf{w}_{i}^{*}\right) \phi \mathrm{d}^{3} r=0
$$

We use divergence-free functions $\mathbf{w}_{\mathbf{i}}$, as demanded by the continuity equation inside the volume, thus the last term in (8) vanishes. The first integral in the last expression is broken into integrals over the two contact surfaces $S_{\text {top }, i}, S_{\text {bot }, i}$ plus the integral over the side walls $S_{\text {side }, i}$ (non-contact surfaces, see Fig.1). On the side walls, we adopt the following choice for $v_{i}$ to describe the surface charge distribution functions:

$$
v_{i} \equiv \mathbf{w}_{\mathbf{i}} \cdot \hat{\mathbf{n}} \quad \text { On side face } S_{i}
$$

resulting in

$$
\begin{aligned}
& \int_{V_{i}} \mathbf{w}_{i}^{*} \cdot \nabla \phi \mathrm{d}^{3} \mathbf{r}+\sum_{\text {sides }} \int_{S_{\text {side }, i}} v_{i}^{*} \phi \mathrm{d}^{2} r= \\
& \int_{S_{t o p, i}}\left(\mathbf{w}_{i}^{*} \cdot \hat{\mathbf{n}}\right) \phi \mathrm{d}^{2} r \quad+\int_{S_{b o t, i}}\left(\mathbf{w}_{i}^{*} \cdot \hat{\mathbf{n}}\right) \phi \mathrm{d}^{2} r
\end{aligned}
$$

The left hand side on this last expression is the sum of the two right hand sides of (4), which we replace by the respective left hand sides.

Consistency between the continuity equation (2) and the choice of functions $v_{i}$ in (9) implies that the values $q_{i}$ in the solution must be such that $j \omega q_{i}=I_{i}$.

As to the rhs of (10), the factors $\mathbf{w}_{\mathbf{i}} \cdot \hat{\mathbf{n}}$ act as weights for the average potential on each contact surface, except for a factor identical to the $z$-dependence $\zeta(z)$ of the $z$ component for the function $\mathbf{w}_{\mathbf{i}}(\mathbf{r})$ and a difference of sign given by the orientation of the nor- mal vectors (see Fig.1). Thus we obtain the following equation for the system:

$$
\begin{array}{r}
{\left[R+j \omega L+\frac{P}{j \omega}\right](I)=(\Delta V)} \\
\text { with }(\Delta V)_{i}=\zeta\left(\ell_{i}\right) V_{f, i}-\zeta(0) V_{0, i}
\end{array}
$$

with the size of the system being $n \times n$. This equation, together with (5) is the basis of our formulation.

\section{CONDUCTION MODES}

The conduction modes are eigenfunctions of the homogeneous vector Helmholtz equation that the current density $\mathbf{J}$ satisfies inside the conductors.

$$
\begin{aligned}
& \left(\nabla^{2}+\kappa^{2}\right) \mathbf{J}(\mathbf{r})=0 \\
& \quad \text { with } \quad \kappa^{2}=-k_{0}^{2}+\frac{j}{\delta^{2}}, \quad \delta=\sqrt{2 / \omega \mu \sigma}
\end{aligned}
$$

This equation is the differential form of (1).

\subsection{RL extraction}

In the MQS version of conduction modes [1], the following assumptions are used:

1. Currents are one dimensional;

2. Current density does not depend on the direction of propagation $(z)$;

3. $\lambda_{\min } \gg \ell$.

In other words:

$$
\begin{aligned}
& \mathbf{J}(\mathbf{r})=J_{z}(x, y) \hat{z} \\
& \phi(\mathbf{r})=\phi(z) \text { for each conductor }
\end{aligned}
$$

The $R L$ conduction mode eigenfunctions are:

$$
\mathbf{w}_{\mathbf{i}}(\mathbf{r})= \begin{cases}\hat{\mathbf{z}} A_{i} e^{-\left[\alpha_{i}\left(x-x_{i}\right)+\beta_{i}\left(y-y_{i}\right)\right]} & \text { if } \mathbf{r} \in V_{n_{i}} \\ 0 & \text { else }\end{cases}
$$

with

$$
\alpha_{i}^{2}+\beta_{i}^{2}=-\left(\frac{2 \pi}{\lambda}\right)^{2}+\left(\frac{1+j}{\delta}\right)^{2}
$$

and $x_{i}, y_{i}$ indicate the corner, on the cross section, from which the conduction mode decays.

Since, $\delta \ll \lambda$, one can neglect the first term in the rhs of (16). For the rhs of (4), the above assumptions lead to:

$$
<\mathbf{w}_{\mathbf{i}}, \nabla \phi>=\int_{V_{i}} \frac{\partial \phi(z)}{\partial z} w_{i}^{*}(x, y) \mathrm{d} \mathbf{r}=\int_{V_{i}} \frac{\partial \phi}{\partial z} \mathrm{~d} z=\phi(L)-\phi(0)
$$

with the $\mathbf{w}_{\mathbf{i}}$ implicit normalization $\int w_{i}(x, y) \mathrm{d} x \mathrm{~d} y=1$ over the cross section of the conductor. The author in [9] found that within this scheme, the inclusion of a few modes suffices for capturing $R L$ effects in the region of small penetration $\delta$. 


\section{2 $R L C$ extraction}

For the coupled fullwave $R L C$ problem, we use eigenfunctions to the vector Helmholtz equation, and since the equation separates in cartesian coordinates the extension is straightforward:

$\mathbf{w}_{\mathbf{i}}(\mathbf{r})= \begin{cases}\left(a_{x i} \hat{\mathbf{x}}+a_{y i} \hat{\mathbf{y}}+a_{z i} \hat{\mathbf{z}}\right) e^{-\left[\alpha_{i}\left(x-x_{i}\right)+\beta_{i}\left(y-y_{i}\right)\right]+\eta_{i}\left(z-z_{i}\right)} & \text { if } \mathbf{r} \in V_{n_{i}} \\ 0 & \text { else }\end{cases}$

with

$$
\alpha_{i}^{2}+\beta_{i}^{2}+\eta_{i}^{2}=-\left(\frac{2 \pi}{\lambda}\right)^{2}+\left(\frac{1+j}{\delta}\right)^{2}
$$

When selecting $\alpha, \beta, \eta$ we must keep the $(2 \pi / \lambda)^{2}$ term in (19) at variance with (16) in the $R L$ case. Our range of lengths is broader than that of the $R L$ counterpart. We associate the $\lambda$ with the $z$ dependence. This particular choice stems from the observation that, for realistic configurations, the wire length is the only length scale that can be comparable to the wavelength, while the wire cross sections, are much smaller than $\lambda$, making $\lambda$-sensitivity in $x$ or $y$ unnoticeable. Moreover, were a matrix element with finite $\eta$ to contain a $\delta$-like dependence, it would lead to an exponentially attenuated function along the $z$-axis, whereas the elements containing no $\delta$ dependence, will only include a phase factor for the $z$ integral. Barring unforeseeable cancellations, our choice is natural. Our decomposition captures the following physical picture: $z$-dependence is that associated with the propagation of electromagnetic waves in the surrounding medium, whereas the $x, y$ dependence represents the re-accomodation of currents inside the conductors, and the deposit of charges on the surfaces, such us to shield the interior from charge accumulation.

Therefore, the choice for $\alpha_{i}, \beta_{i}$ remains as in (16), while for $\eta$ we have:

$$
\eta_{i}= \pm j \frac{2 \pi}{\lambda}
$$

where the sign assignment corresponds to the direction of propagation either going up or down along the $z$ axis.

Due to (2a), the coefficients must satisfy

$$
a_{x i} \alpha_{i}+a_{y i} \beta_{i}+a_{z i} \eta_{i}=0
$$

This last condition restricts the spatial direction for basis functions $\mathbf{w}_{\mathbf{i}}$

$$
\left(a_{x i}, a_{y i}, a_{z i}\right)=A_{i}\left(\frac{1}{\alpha_{i}}, \frac{1}{\beta_{i}},-\frac{2}{\eta_{i}}\right)+B_{i}\left(\frac{1}{\alpha_{i}},-\frac{1}{\beta_{i}}, 0\right)
$$

for arbitrary $A_{i}, B_{i}$ nonzero complex numbers.

The direction of the second vector in the rhs of (22) represents solutions with no current flow along the $z$ axis, direction of the applied gradient of the potential. These solutions carry no net charge, and represent redistribution of currents along the cross section of a conductor. We neglect their contribution, and keep only a vector that is perpendicular to it. We thus determine the values $A_{i}$ and $B_{i}$, up to an arbitrary normalization constant.

We choose to normalize the basis functions such that the total current entering the conductor at the bottom face can be represented as the sum of all the coefficients in (3b) corresponding to that conductor $I_{\text {conductor } k}=\sum_{i \in k} I_{i}$.

This choice fixes the arbitrary constant remaining in (22) :

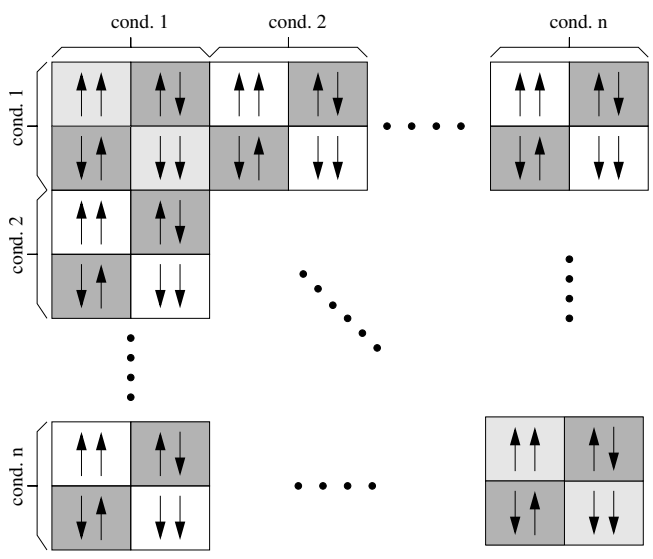

Figure 2: Structure of the matrix in (11) according to the propagation in $z$ (arrows). The darker boxes represent larger elements in that part of the matrix; the diagonal elements contain the contribution of the $R$ elements.

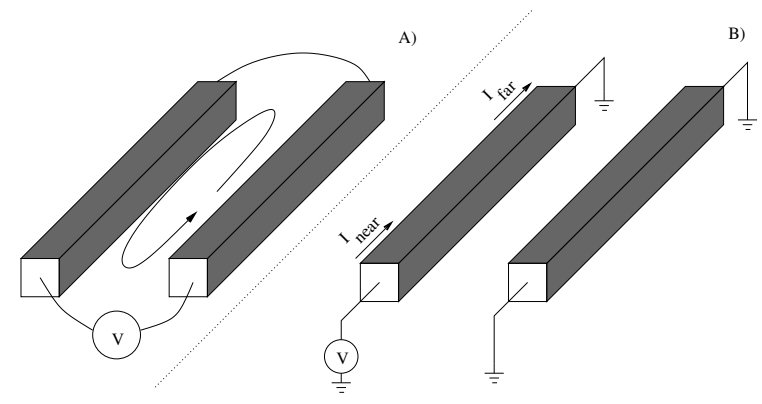

Figure 3: Example configurations for: (a) $R L$ case; (b) $R L C$ case.

$$
\left(a_{x i}, a_{y i}, a_{z i}\right)=\left(\frac{\alpha_{i}}{e^{\alpha_{i} d_{i}}-1}\right)\left(\frac{\beta_{i}}{e^{\beta_{i} h_{i}}-1}\right)\left(\delta^{2} \alpha_{i} \eta_{i}, \delta^{2} \beta_{i} \eta_{i}, 1\right)
$$

with $d_{i}$ and $h_{i}$, the width and thickness of the conductor, respectively. In general, a conduction mode will have both $\alpha_{i}, \beta_{i} \neq 0$, and we will refer to it as a corner mode. If one of these parameters is null, we will call it a side mode. If $\alpha_{i}$ is null, then the first fraction in the expression above should be replaced by $d_{i}^{-1}$, likewise, the second fraction is replaced by $h_{i}^{-1}\left(\alpha_{i}\right.$ and $\beta_{i}$ cannot both be null due to (16)).

For every current conduction mode, there is a corresponding surface charge function $v_{i}$ computed using (9).

\section{IMPLEMENTATION}

Our next task is to compute the matrix elements in (5), and solve (11) to obtain the currents on all the conductors. Computation of the matrix elements is nontrivial.

The $R_{i j}$ elements pose no problem, the three dimensional overlap integrals can be computed analytically, they are only different from zero for $i, j$ running over the same conductor, the result is: 


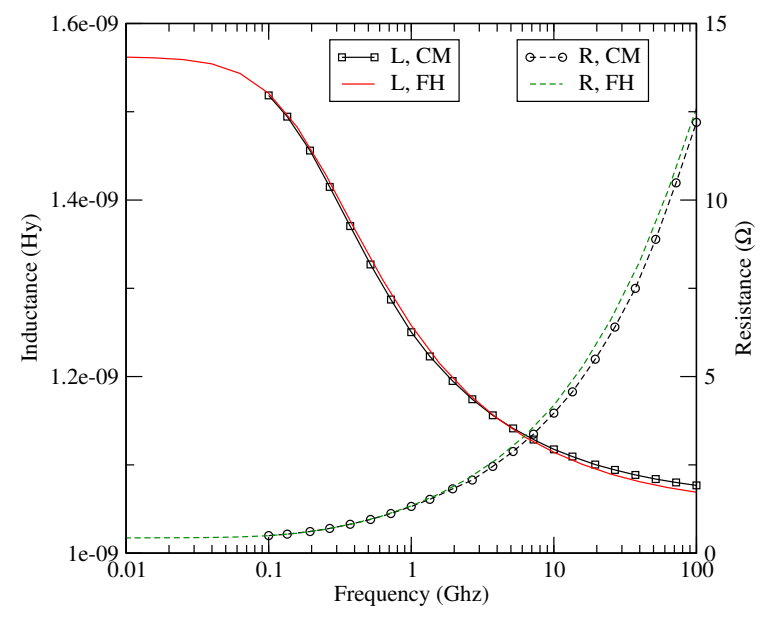

Figure 4: Conduction Modes (CM) and FastHenry (FH) results for loop resistance and inductance, example Fig.3a over a wide frequency range.

$$
R_{i j}=\delta_{n_{i} n_{j}} A_{i}^{*} A_{j} \frac{\left(e^{\left(\alpha_{i}^{*}+\alpha_{j}\right) d_{i}}-1\right)\left(e^{\left(\beta_{i}^{*}+\beta_{j}\right) h_{i}}-1\right)\left(e^{\left(\eta_{i}^{*}+\eta_{j}\right) l_{i}}-1\right)}{\left(\alpha_{i}^{*}+\alpha_{j}\right)\left(\beta_{i}^{*}+\beta_{j}\right)\left(\eta_{i}^{*}+\eta_{j}\right)}
$$

At high frequencies the conduction modes become very localized, rendering $R$ matrix nearly diagonal.

The six dimensional $L$ and the four dimensional $P$ integrals (5) demand special attention. As a matter of fact at least three dimensions in the six dimensional integral in $L$ can be evaluated analytically, similarly two out of the four dimensions in the $P$ evaluation can also be evaluated analytically.

We noticed the following property: the capacitive $(P)$ contribution nearly cancels out with the inductive $(L)$ one for the case where the two modes $i, j$ correspond to waves traveling in the same sense on the $z$-direction (in other words, when $\eta_{i}=\eta_{j}$ ), and give nearly the same when the modes are opposing waves in $z\left(\eta_{i}=-\eta_{j}\right)$.

This is strictly a high frequency phenomenon. In fact, in the (infinite-frequency) limit $\delta \rightarrow 0$, the $L$ and $P$ contributions with $\eta_{i}=\eta_{j}$ cancel exactly each other out. The origin of this near cancellation and doubling stems from the observation that, after some algebra, the $L$ contribution for these modes may be written as

$$
L=\iint_{y, z}\left(\int_{0}^{d} e^{-x / \delta} f(x, y, z) \mathrm{d} x\right) \mathrm{d} y \mathrm{~d} z
$$

whereas the $P$ contribution becomes:

$$
P / \omega^{2}= \pm \iint_{y, z} f(0, y, z) \mathrm{d} y \mathrm{~d} z
$$

both with the same integrand function $f(x, y, z)$. In other words, the $P$ matrix elements, divided by $\omega^{2}$, are the collapsed version of the $L$ ones, up to a sign according to whether the element correspond to two modes traveling in the same or opposite sense along the $z$-axis.

Given this state of affairs, we always pair up the combination $j \omega\left(L_{i j}-P_{i j} / \omega^{2}\right)$ together (see (11)). Otherwise, we would be incurring in large numerical errors by computing them separately and canceling out two similar magnitudes, each with a certain numerical error.

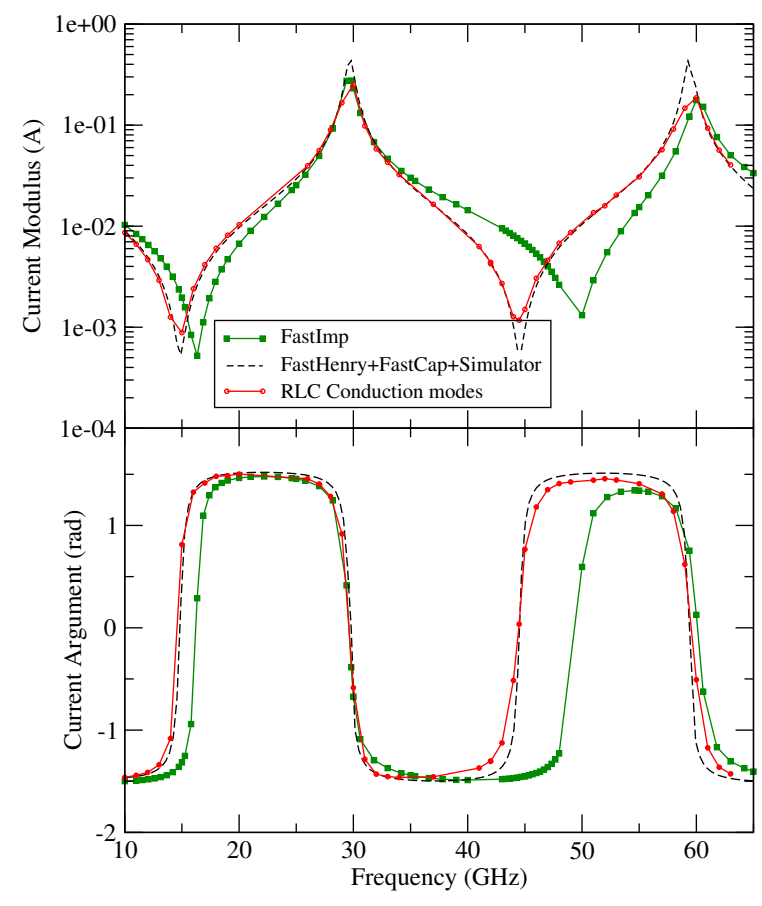

Figure 5: $R L C$ results, for the example in Fig.3b: modulus (top) and argument (bottom) for the aggressor's near end current $I_{\text {near }}$ for Conduction Modes and other methods described in text.

The matrix $Z=R+j \omega\left(L-P / \omega^{2}\right)$ in (11), has a checker-board structure as shown in Fig.2. This alternation between small and large elements while decomposing into forward- and backwardtraveling waves, is responsible for the appearance of marked resonance peaks observed in the following section.

The numerical computation of the integrals is done with a combination of Monte Carlo, Gaussian quadrature, and Taylor series expansion following standard schemes [10].

We are testing small dimensionality problems, for which we use Singular Value Decomposition method $[10,11]$ to solve the system matrix, equation (11).

\section{EXAMPLES}

To calibrate our expectations we start by evaluating the matrix elements in the $R L \mathrm{MQS}$ regime. We select a problem such that the skin effect is important. To this end, we neglect the $x-$ and $y-$ components of the current $\mathbf{J}$ in (22) and set $k_{0}=0$. There is no $z-$ dependence, therefore there is no distinction between backwardand forward-traveling waves. Our example is drawn from [12], consisting of two $\mathrm{Cu}$ wires $5 \mathrm{~mm}$ long, of cross sections $10 \mu \mathrm{m} \times$ $40 \mu m$, separated by a distance of $10 \mu m$, shorted at the far end (see Fig.3a). In Fig.4 we compare the loop inductance and resistance to the $R L$ standard solver FastHenry. There is total agreement. Four side modes per conductor sufficed to get this level of agreement. It should be noted that the numerical computation of $L_{i, j}$ is two dimensional in the $R L$ regime, rather than three dimensional as in the general $R L C$ case.

We consider next the fullwave regime. We take the same geometrical example, grounding all terminals except one where the input excitation is introduced (Fig.3b). We use the full wave representation for the matrix elements, with 16 modes per conductor: 4 side plus 4 corner for each possible $z$ - propagation. In Figs. 5 and 


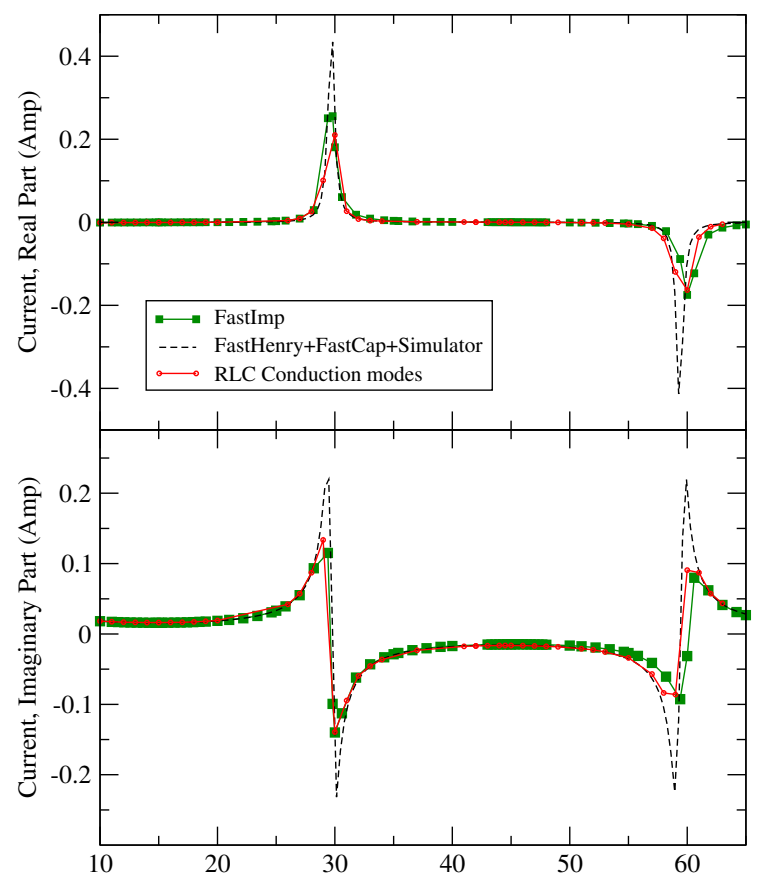

Figure 6: $R L C$ results, for the example in Fig.3b: real (top) and imaginary (bottom) parts for the aggressor's far end current $I_{\text {far }}$. for Conduction Modes and other methods described in text.

6 we compare the currents obtained at the near and far end of the aggressor against:

- FastImp, used in the fullwave mode [5]

- Spice simulation using transmission-line model with parameters obtained with the help of FastHenry and FastCap.

We observe resonance peaks at the right frequencies multiples of quarter wave lengths. Qualitatively, the merged $R L C$ volume solver shows the correct physical behavior. The location of the minima is different on FastImp compared to our's as well as the transmission line model. The exact origin of this discrepancy is not yet completely understood.

Further development is ongoing, both to increase our understanding of the algorithmic advantages and or pitfalls of the method. Among them, the "intelligent" choice of modes according to the particular geometry of the problem at hand. The acceleration of the numerical integration schemes comes next in our long list of priorities.

We rest quite hopeful regarding the advantages of the method, given that for these simple problems at least, the dimensionality of the resulting linear system is $n=64$ dimensions on conduction modes, compared to $n=4612$ on FastImp, and $n=1936$ for FastHenry plus $n=136$ for FastCap, a sensible improvement. Furthermore, the convergence to FastCap plus FastHenry in the low frequency regime with a fairly simplified description is equally rewarding.

\section{CONCLUSIONS}

We presented the essence of a new simulation approach to study $R L C$ fullwave modeling of conductors in terms of a small set of conduction basis functions. We have been able to capture the interplay between the electric and magnetic energy content that gives rise to resonances, while keeping a very concise description of the geometry. Our model resembles a transverse electromagnetic (TEM) model with frequency dependent parameters.

We have omitted the extensive algebra associated with the detailed computation of the matrix elements, algebra that we leave for a more detailed version of this work. We have several challenges ahead of us to arrive to computationally inexpensive recipes. We plan to thoroughly explore different choices of conduction modes in terms of the extraction results so as to systematize the choice of conduction modes as a function of problem geometry. Next step is to address different accuracy vs. performance ratios in the computation of the numerical integrals.

\section{REFERENCES}

[1] L. Daniel, A. Sangiovanni-Vincentelli, and J. White, "Using conduction modes basis functions for efficient electromagnetic analysis of on-chip and off-chip interconnect," in Design Automation Conference (DAC), 2001. Proceedings, 2001, pp. 563-566.

[2] W. C. Chew, J.-M. Jin, E. Michielssen, and J. S. (Eds.), Fast and efficient algorithms in computational electromagnetics. Boston: Arter House, Inc., 2001.

[3] K. Nabors and J. White, "Fastcap: a multipole accelerated 3-d capacitance extraction program," vol. 10, no. 11, pp. 1441-1459, 1991.

[4] M. Kamon, M. J. Tsuk, and J. White, "Fasthenry: A multipole-accelerated 3-d inductance extraction program," IEEE Trans. Microwave Theory Tech., vol. 42, no. 9, pp. 1750-1758, Sept. 1994.

[5] Z. Zhu, B. Song, and J. White, "Algorithms in fastimp: a fast and wide-band impedance extraction program for complicated 3-d geometries," vol. 24, pp. 981-998, July 2005.

[6] J.-S. Zhao and W. C. Chew, "Integral equation solution of Maxwell's equations from zero frequency to microwave frequencies," in Antennas and Propagation, IEEE Transactions on, vol. 48, Oct 2000, pp. 1635-1645.

[7] X. Hu, J. H. Lee, L. Daniel, and J. White, "Analysis of full-wave conductor system impedance over substrate using novel integration techniques," in Design Automation Conference (DAC), 2005. Proceedings, 2005, pp. 147-152.

[8] J. Jackson, Classical Electrodynamics. New York: John Wiley and Sons, Inc., 1962.

[9] L. Daniel, "Simulation and modeling techinques for signal integrity and electromagnetic interference on high frequency electronic systems," Ph.D. dissertation, University of California at Berkeley, 2003.

[10] W. H. Press, B. P. Flannery, S. A. Teukolsky, and W. T. Vetterling, Numerical Recipes: The Art of Scientific Computing, 1st ed. Cambridge (UK) and New York: Cambridge University Press, 1986.

[11] G. Golub and C. van Loan, Matrix Computations, 3rd Ed. Baltimore: The Johns Hopkins University Press, 1992.

[12] X. Hu, L. Daniel, and J. White, "Partitioned conduction modes in surface integral equation-based impedance extraction," in Electrical Performance of Electronic Packaging, Oct 2003, pp. 355-358. 